\title{
HOMOGENIZATION OF THE BACKWARD-FORWARD MEAN-FIELD GAMES SYSTEMS IN PERIODIC ENVIRONMENTS
}

\author{
PIERRE-LOUIS LIONS ${ }^{1}$ AND PANAGIOTIS E. SOUGANIDIS ${ }^{2,3}$
}

Wednesday $11^{\text {th }}$ September, 2019

\begin{abstract}
We study the homogenization properties in the small viscosity limit and in periodic environments of the (viscous) backward-forward mean-field games system. We consider separated Hamiltonians and provide results for systems with (i) "smoothing" coupling and general initial and terminal data, and (ii) with "local coupling" but well-prepared data.The limit is a first-order forward-backward system. In the nonlocal coupling case, the averaged system is of mfg-type, which is well-posed in some cases. For the problems with local coupling, the homogenization result is proved assuming that the formally obtained limit system has smooth solutions with well prepared initial and terminal data. It is also shown, using a very general example (potential $\mathrm{mfg}$ ), that the limit system is not necessarily of mfg-type.
\end{abstract}

Key words and phrases mean-field games, back-forward systems, periodic homogenization, small noise limit, ergodic problem, two-scale weak convergence

AMS Class. Numbers 35B27, 35B40, 35K40, 35K59, 91A13

\section{Introduction}

This paper is the first step of a general program to study the homogenizing properties of mean-field games (for short $\mathrm{mfg}$ ) set in self-averaging, for example, periodic or stationary ergodic environments.

In particular, we study the homogenization properties, in periodic environments, of the classical viscous backward-forward mfg-system

$$
\left\{\begin{array}{l}
\partial_{t} u^{\epsilon}-\epsilon \Delta u^{\epsilon}+H\left(D u^{\epsilon}, \frac{x}{\epsilon}\right)-F_{\epsilon}\left[m^{\epsilon}\right]=0 \text { in } \mathbb{R}^{d} \times(0, T) \quad u^{\epsilon}(\cdot, 0)=u_{0}, \\
\partial_{t} m^{\epsilon}+\epsilon \Delta m^{\epsilon}+\operatorname{div}\left[D_{p} H\left(D u^{\epsilon}, \frac{x}{\epsilon}\right) m^{\epsilon}\right]=0 \text { in } \mathbb{R}^{d} \times(0, \infty) \quad m^{\epsilon}(\cdot, T)=m_{T} .
\end{array}\right.
$$

which is the core of the mean field games theory without common noise.

In (1.1), $H=H(p, y): \mathbb{R}^{d} \times \mathbb{R}^{d} \rightarrow \mathbb{R}$ is periodic in the second argument on the torus $\mathbb{T}^{d}$ and $D_{p} H$ is the derivative of $H$ with respect to its first argument.

We discuss next the forcing term $F_{\epsilon}$ in (1.1). To present a unified setting, that is, to discuss at the same time both the cases of local and nonlocal (smoothing) dependence on the density, we assume that

where

$$
F_{\epsilon}[m](x)=F\left(x, \frac{x}{\epsilon}, m\right)
$$

$$
F=F(x, y, m): \mathbb{R}^{d} \times \mathbb{R}^{d} \times C^{2}\left(\mathbb{R}^{d}\right) \rightarrow \mathbb{R} \text { is } \mathbb{T}^{d} \text {-periodic in } y .
$$

When dealing with the local case, (1.3) follows from assuming that

$$
F=F(x, y, m): R^{d} \times \mathbb{R}^{d} \times \mathbb{R} \rightarrow \mathbb{R} \text { is } \mathbb{T}^{d} \text {-periodic in } y,
$$


in which case

$$
F_{\epsilon}[m](x)=F\left(x, \frac{x}{\epsilon}, m(x)\right) .
$$

When we consider the nonlocal dependence on the density, for simplicity, we will omit the $y$-dependence of $F$ in (1.3) and extend the domain of $F$ in (1.3), that is, we assume that

$$
F=F(x, m): \mathbb{R}^{d} \times \mathcal{P} \rightarrow \mathbb{R},
$$

where $\mathcal{P}$ is the space of probability measures on $\mathbb{R}^{d}$.

Most of the results extend to mfg-systems with not separated Hamiltonians, that is $H$ that also depend on $m$. To keep, however, the notation and to explain the main ideas in this note we chose to work with (1.1).

Our main results are: (i) Homogenization to a unique limit (1.1) with nonlocal coupling and general data to an mfg-system. (ii) Homogenization for (1.1) with local coupling to a forward-backward system, when the latter has a smooth solution and (1.1) has well-prepared data. The limit system is not, however, in general of mfg-type.

In either case, the classical ansatz

$$
u^{\epsilon}(x, t)=\bar{u}(x, t)+\epsilon v\left(\frac{x}{\epsilon}\right) \text { and } m^{\epsilon}(x, t)=\bar{m}(x, t)\left(\mu\left(\frac{x}{\epsilon}\right)+\epsilon \nu\left(\frac{x}{\epsilon}\right)\right)
$$

with

$$
v, \mu, \nu: \mathbb{R}^{d} \rightarrow \mathbb{R} \text { are } \mathbb{T}^{d} \text { - periodic, } \mu>0 \text { and } \int_{\mathbb{R}^{d}} \mu(y) d y=1,
$$

formally leads, for each $p \in \mathbb{R}^{d}$ and $m \in L^{1}\left(\mathbb{R}^{d}\right)$ or $m \in \mathbb{R}$, to the mfg-ergodic system (cell-problem)

$$
\left\{\begin{array}{l}
-\Delta v+H(D v+p, y)-F[m \mu]=\bar{H}(p, m, x) \text { in } \mathbb{T}^{d}, \\
\Delta \mu+\operatorname{div}\left[D_{p} H(D v+p, y) \mu\right]=0 \text { in } \mathbb{T}^{d}, \\
\int_{\mathbb{T}^{d}} v(y) d y=0, \mu>0 \text { in } \mathbb{T}^{d} \text { and } \int_{\mathbb{T}^{d}} \mu(y) d y=1,
\end{array}\right.
$$

where $y=x / \epsilon$ and $F[m \mu]$ is defined as in (1.2), (1.3) with $\epsilon=1$. Note that the dependence of $\bar{H}$ on $x$ is through $F$.

It is known (see Lasry and Lions [23], Lions [24], and Cardaliaguet, Lasry, Lions and Porretta [5, 6]) that, under the appropriate conditions on $H$ and $F$ to be introduced later, for each $x, p \in \mathbb{R}^{d}$ and $m: \mathbb{R}^{d} \rightarrow \mathbb{R}$ smooth, (4.9), there exists a unique constant $\bar{H}(p, x, m)$ such that (3.5) has a unique $\mathbb{T}^{d}$-periodic solution $(v, m)=(v(\cdot ; p, x, m), \mu(\cdot ; p, x, m)) \in C^{2}\left(\mathbb{R}^{d}\right) \times L^{1}\left(\mathbb{R}^{d}\right)$.

The homogenized system is then

$$
\left\{\begin{array}{l}
\partial_{t} \bar{u}+\bar{H}(D \bar{u}, x, \bar{m})=0 \text { in } \mathbb{R}^{d} \times(0, T] \quad \bar{u}(\cdot, 0)=u_{0}, \\
\partial_{t} \bar{m}+\operatorname{div}[\bar{b}(D \bar{u}, x, \bar{m}) \bar{m}]=0 \text { in } \mathbb{R}^{d} \times[0, T) \quad \bar{m}(\cdot, T)=m_{T},
\end{array}\right.
$$

where, for $p \in \mathbb{R}^{d}$ and $m \in L^{1}\left(\mathbb{R}^{d}\right)$ or $m \in \mathbb{R}$,

$$
\bar{b}(p, x, m)=\int_{\mathbb{T}^{d}} D_{p} H(p+D v(y ; p, x, m), y) \mu(y ; p, x, m) d y .
$$

An important question is whether (1.10) is actually an mfg-system, that is, whether, for all $p \in \mathbb{R}^{d}$ and $m \in L^{1}\left(\mathbb{R}^{d}\right)$ or $m \in \mathbb{R}$,

$$
\bar{b}(p, x, m)=D_{p} \bar{H}(p, x, m) .
$$

We state now in an informal way, that is, without precise assumptions, the two main convergence results. The first is about the nonlocal setting and the second about the local one. 
Theorem 1.1. Assume that (1.1) is nonlocal, that is, $F_{\epsilon}$ is given by (1.5) and (1.6). Then, along subsequences $\epsilon \rightarrow 0$, locally uniformly in $\mathbb{R}^{d} \times[0, T]$ and in $L^{1}\left(\mathbb{R}^{d} \times(0, T)\right)$, the solution $\left(u^{\epsilon}, m^{\epsilon}\right)$ of $(1.1)$ converges to a solution $(\bar{u}, \bar{m})$ of (1.10), which is always of mfg-type. If, in addition, (1.1) satisfies the conditions that make it well-posed, then (1.10) is also well-posed and the full family converges to the unique solution of (1.10).

Theorem 1.2. Assume that the coupling in (1.1) is local, that is, $F_{\epsilon}$ satisfies (1.2) and (1.3). If (1.10) has a smooth solution $(\bar{u}, \bar{m})$ and (1.1) has well prepared initial and terminal data, then, as $\epsilon \rightarrow 0$, locally uniformly in $\mathbb{R}^{d} \times[0, T]$ and in $L^{1}\left(\mathbb{R}^{d} \times(0, T)\right)$, the solution $\left(u^{\epsilon}, m^{\epsilon}\right)$ of $(1.1)$ converges to $(\bar{u}, \bar{m})$. In general, (1.10) is not an mfg-type system.

A particular case of Theorem 1.2 is when, for some $p \in \mathbb{R}^{d}$,

$$
u_{0}(x)=(p, x) \text { and } m_{T} \equiv 1 \text {. }
$$

In this case, $(\bar{u}, \bar{m})$ with $\bar{u}(x, t)=(p, x)-t \bar{H}(p, 1)$ and $m \equiv 1$ solves (1.10), and an argument similar to the proof of Theorem 2.18 gives that, as $\epsilon \rightarrow 0$ and locally uniformly in $\mathbb{R}^{d} \times[0, T]$ and in $L^{1}\left(\mathbb{R}^{d} \times(0, T)\right)$,

$$
u^{\epsilon}(x, t) \rightarrow(p, x)-\bar{H}(p, 1) \text { and } m^{\epsilon} \rightarrow 1 .
$$

Finally, we note that after the results of this paper were obtained and announced, the authors became aware of a work by Cesaroni, Dirr and Marchi [10] which is about a special class of (1.1) with quadratic $H$ and set in $\mathbb{T}^{d} \times[0, T]$.

Background. The theory of mean-field games was introduced about a decade ago with by Lasry and Lions [23], who developed the fundamental elements of the mathematical theory, and, independently, by Huang, Malhamé, and Caines [19] who considered a particular class of mfg. Since then, the subject has grown rapidly both in terms of theory and applications. Some of the landmark theoretical results include the introduction of the so-called "Master equation," the theory of "monotone hyperbolic systems" for finite state space models, and the "Hilbertian" approach for infinite dimensional problems. References for these developments are the courses of Lions at College de France [24], which are available on line, and the forthcoming books of Carmona and Delarue [8, 9]. A partial and by no means complete list of references of the earlier work in this general area includes (in alphabetical order) Achdou, Buera, Lasry, Lions and Moll [1], Achdou, Giraud, Lasry and Lions [2], Cardalliaguet, Delarue, Lasry and Lions [4], Cardaliaguet, Lasry, Lions and Porretta [5, 6], Carmona and Delarue [7], Gabaix, Lasry, Lions and Moll [12], Guéant, Lasry and Lions [14], Huang, Caines and Malhamé [18], Huang, Malhamé, and Caines [15, 17, 16], Lachapelle, Lehalle, Lasry and Lions [20], and Lasry and Lions [21, 22, 25].

Applications that have been so far looked at range from complex socio-economical topics, regulatory financial issues, crowd movement, meaningful "big" data and advertising to engineering contexts involving "decentralized intelligence" and machine learning; two concrete examples being fleets of automated cars and future telecommunication networks.

At the beginning, MFG models were introduced to describe the behavior of a large group or several groups of agents using a mean field approach as in statistical physics. Such models can be derived rigorously from $N$-players systems as $N$ tends to infinity. In this context, an agent is someone trying to optimize certain criteria which, together with its dynamics, depend on the other agents and their actions. It is important to emphasize that the agents react, anticipate and strategize instead of simply reacting instantaneously. The latter is, for example, the case in many agent-based models or ones derived from statistical mechanics and/or kinetic considerations.

MFG are the ideal mathematical structures to study the quintessential problems in the social-economical sciences, which differ from physical settings because of the forward looking behavior on the part of 
individual agents. Concrete examples of applications in this direction include the modeling of the macroeconomy and conflicts in the modern era. In both cases, a large number of agents interact strategically in a stochastically evolving environment, all responding to partly common and partly idiosyncratic incentives, and all trying to simultaneously forecast the decision of others.

Future work. This note is the first in a series of works that will provide a systematic homogenization theory for mean-field games. Among others, we will consider general initial and terminal data for (1.1), we will study the well-posedness of the limit system when it is not of mfg-type, and, finally, we will consider extensions to random media as well as to $\mathrm{mfg}$ with variational structure and even common noise.

Organization of the paper. In section 2 we discuss the ansatz, we derive formally both the cell problem and the homogenized systems, and state the result about the solvability of the cell problem. In section 3 we concentrate on the nonlocal problem. In section 4 , we study the local problem. We show that it homogenizes to a system which is not always of mfg-type.

Notation. Throughout the paper $C_{c}\left(\mathbb{R}^{d}\right)$ denotes the space of compactly supported continuous functions on $\mathbb{R}^{d}$. When more regularity is needed, we simply say smooth functions in $C_{c}\left(\mathbb{R}^{d}\right)$. We also write $C_{c, p}\left(\mathbb{R}^{d} \times \mathbb{T}^{d}\right)$ for the space of continuous functions which are compactly supported in the first argument and $\mathbb{T}^{d}$-periodic in the second, and we use the same convention when more regularity is needed. Finally, $C_{b}(U ; V)$ is the space of continuous bounded functions defined on $U$ and values in $V$.

Acknowledgment. The first author was partially supported by the Air Force Office for Scientific Research grant FA9550-18-1-0494 and the Office for Naval Research grant N000141712095. smallskip The second author was partially supported by the National Science Foundation grant DMS-1600129, the Office for Naval Research grant N000141712095 and the Air Force Office for Scientific Research grant FA9550-18-1-0494.

\section{The Assumptions}

As far as $H: \mathbb{R}^{d} \times \mathbb{R}^{d} \rightarrow \mathbb{R}$ is concerned, throughout the paper we assume that

$$
\begin{gathered}
y \rightarrow H(p, y) \text { is periodic in } \mathbb{T}^{d} \text { for each } p \in \mathbb{R}^{d}, \\
p \rightarrow H(p, y) \text { is uniformly convex in } p \text { for each } y \in \mathbb{T}^{d},
\end{gathered}
$$

and

$$
H \text { and } D_{p} H \text { are uniformly bounded in } y \text { for } p \text { bounded in } \mathbb{R}^{d} \text {; }
$$

The assumptions on $F$ and the joint dependence of $H$ and $F$ in $x$ and $y$ depend on the type of the coupling we consider.

The nonlocal coupling. Throughout the discussion of the mfg-systems with nonlocal coupling, we assume that the coupling $F_{\epsilon}$ is independent of $\epsilon$, that is

$$
F_{\epsilon}[m](x)=F[m](x)=F(x, m)
$$

where $F: \mathbb{R}^{d} \times \mathcal{P} \rightarrow \mathbb{R}$ is

$$
x \rightarrow F(x, m) \text { is Lipschitz continuous uniformly in } x \in \mathbb{R}^{d} \text { and } m \in \mathcal{P},
$$

and

$m \rightarrow F(x, m)$ is continuous with respect to the weak topology of measures uniformly in $x$ that is,

$$
\text { if } m^{\epsilon} \underset{\epsilon \rightarrow 0}{\rightarrow} m \text { in the sense of measures, then, uniformly in } x, F\left(x, m^{\epsilon}\right) \underset{\epsilon \rightarrow 0}{\rightarrow} F(x, m) .
$$


In order to obtain gradient bounds for $u^{\epsilon}$ in (1.1), it is necessary to assume some additional conditions on $H$ and $F$. As far as the latter is concerned, we assume that

$$
m \rightarrow F[m] \text { is differentiable in } m \text { and } F^{\prime}[m] \text { is smooth and bounded in } \mathcal{P}
$$

that is,

$$
\left\|D_{x} F^{\prime}[m]\right\|_{\infty}+\left\|D_{x}^{2} F^{\prime}[m]\right\|_{\infty} \text { is bounded in } \mathcal{P} .
$$

We also need to assume that

$$
\left\{\begin{array}{l}
\text { there exists } \theta \in(0,1) \text { such that, for } \epsilon \in(0,1), \text { large }|p| \text { and } m \in \mathcal{P}, \\
\inf _{x \in \mathbb{R}^{d}, y \in \mathbb{T}^{d}}\left\{\theta H^{2}(p, y)+d\left(D_{y} H(p, y), p\right)-\epsilon d\left(D_{x} F(x, m), p\right)\right\}>0 .
\end{array}\right.
$$

As far as the well-posedness of (1.1) goes, it was shown in [23, 24] that (1.1) has a unique solution, if, either

that is,

$$
m \rightarrow F[m] \text { is monotone in } \mathcal{P}
$$

$$
\int_{\mathbb{R}^{d}}\left(F\left(x, m_{1}\right)-F\left(x, m_{2}\right)\right)\left(m_{1}(x)-m_{2}(x)\right) d x \geq 0 \text { for all } m_{1}, m_{2} \in \mathcal{P},
$$

and

$$
H \text { is strictly convex in } p \text { uniformly in } y \in \mathbb{T}^{d} \text {, }
$$

that is,

$$
\text { if } H(p+q, y)-H(p, y)-\left(D_{p} H(p, y), q\right)=0 \text {, then } q=p \text {, }
$$

or

that is,

$$
F \text { is strictly monotone in } \mathcal{P} \text {, }
$$

$$
\text { if } \int_{\mathbb{R}^{d}}\left(F\left(x, m_{1}\right)-F\left(x, m_{2}\right)\right)\left(m_{1}-m_{2}\right)(x) d x=0, \text { then } m_{1}=m_{2} .
$$

The local coupling. When the coupling is local, that is

$$
F_{\epsilon}[m](x)=F\left(x, \frac{x}{\epsilon}, m(x)\right),
$$

with $F$ as in (1.4) and

$$
F \in C^{2}\left(\mathbb{R}^{d} \times \mathbb{R}^{d} \times \mathbb{R}\right) .
$$

Since for the result we assume that the formally derived limiting system has smooth we do not need uniform in $\epsilon$ Lipschitz bounds on $u^{\epsilon}$, and, hence, assumptions like (2.8), (2.8) and (2.10).

For the well-posedness of (1.1), it is necessary, as in the nonlocal case, to assume that

$$
H \text { is strictly convex and, for all } \epsilon>0
$$

and $F_{\epsilon}$ is strictly monotone, that is, there exists $c>0$ such that, for each $\epsilon>0$ and any smooth functions $m_{1}, m_{2}$,

$$
\int_{\mathbb{R}^{d}}\left(F_{\epsilon}\left[m_{1}\right](x)-F_{\epsilon}\left[m_{2}\right](x)\right)\left(m_{1}(x)-m_{2}(x)\right) d x \geq c \int_{\mathbb{R}^{d}}\left|m_{1}(x)-m_{2}(x)\right| d x .
$$

In view of the form of $F_{\epsilon}$, we explain next a condition that $F$ in (1.4) must satisfy in order for $F_{\epsilon}$ to be monotone in the sense of (2.18). For simplicity we assume that $c=0$ in (2.18).

Recall that $F_{\epsilon}$ is monotone for each fixed $\epsilon>0$, if, for all smooth $f_{1}, f_{2}: \mathbb{R}^{d} \rightarrow \mathbb{R}$,

$$
\int_{\mathbb{R}^{d}}\left(F\left(x, \frac{x}{\epsilon}, f_{1}(x)\right)-F\left(x, \frac{x}{\epsilon}, f_{2}(x)\right)\right)\left(f_{1}(x)-f_{2}(x)\right) d x \geq 0 .
$$


A trivial approximation argument then yields that (2.19) implies that, for each $\epsilon>0$, and all balls $B \subset \mathbb{R}^{d}$ and $f_{1}, f_{2}: \mathbb{R}^{d} \rightarrow \mathbb{R}$ smooth and $\mathbb{T}^{d}$-periodic,

$$
\int_{\mathbb{R}^{d}}\left(F\left(x, \frac{x}{\epsilon}, f_{1}\left(\frac{x}{\epsilon}\right) \chi_{B}(x)\right)-F\left(x, \frac{x}{\epsilon}, f_{2}\left(\frac{x}{\epsilon}\right) \chi_{B}(x)\right)\right)\left(f_{1}\left(\frac{x}{\epsilon}\right)-f_{2}\left(\frac{x}{\epsilon}\right)\right) \chi_{B}(x) d x \geq 0,
$$

where $\chi_{B}$ is the characteristic function of $B$.

It follows, after letting $\epsilon \rightarrow 0$, that, for all balls $B$ in $\mathbb{R}^{d}$ and $f_{1}, f_{2}: \mathbb{R}^{d} \rightarrow \mathbb{R}$ smooth and $\mathbb{T}^{d}$-periodic, that $F$ in (1.4) must satisfy

$$
\int_{B} \int_{\mathbb{T}^{d}}\left(F\left(x, y, f_{1}(y)\right)-\left(x, y, f_{2}(y)\right)\left(f_{1}(y)-f_{2}(y)\right) d y d x \geq 0,\right.
$$

and, hence, for all $x \in \mathbb{R}^{d}$ and $\left.f_{1}, f_{2}: \mathbb{R}^{d} \rightarrow \mathbb{R}\right)$ smooth and $\mathbb{T}^{d}$-periodic,

$$
\int_{\mathbb{T}^{d}}\left(F\left(x, y, f_{1}(y)\right)-\left(x, y, f_{2}(y)\right)\left(f_{1}(y)-f_{2}(y)\right) d y d x \geq 0 .\right.
$$

\section{The MFG-CEll Problem AND Derivation of the AVERAGEd System}

The aim here is to use the ansatz (1.7) to derive formally the mfg-cell problem (4.9). For its rigorous analysis, that is, existence of ergodic constant and correctors, under the assumptions we introduce in the next two sections, we refer to [23, 24].

The formal argument relies on combining facts from the theory of the homogenization of viscosity solutions and the two scale convergence-see Lions, Papanicolaou and Varadhan [26]), Allaire [3], Nguetseng [28] and Goudon and Poupaud [13].

Inserting the assumed expansion for $u^{\epsilon}$ in the first equation of (1.1), expanding in $\epsilon$, writing $y$ for $x / \epsilon$ and recording only the $\epsilon^{0}$-order term in the formal expansion in powers of $\epsilon$ leads to

$$
\partial_{t} \bar{u}-\Delta_{y} v+H\left(D_{x} \bar{u}+D_{y} v, y\right)-F(x, y, \bar{m} \mu)=0
$$

and, hence, to

$$
\partial_{t} \bar{u}+\bar{H}(D \bar{u}, \bar{m}, x)=0
$$

where, for each $p \in \mathbb{R}^{d}$ and $\bar{m} \in R, v=v(y ; p, \bar{m})$ and $\mu=\mu(y ; p, \bar{m})$,

$$
-\Delta v+H(p+D v, y)-F(x, y, \bar{m} \mu)=\bar{H}(p, \bar{m}, x) .
$$

Note that although the dependence in $p$ and $m$ is separated in the cell problem, the effective Hamiltonian is not necessarily separated.

Inserting the expansion for $m^{\epsilon}$ in the second equation of (1.1), and again "expanding" in powers of $\epsilon$ yields and using the previous notation we find as coefficients of $\epsilon^{-1}$ and $\epsilon^{0}$ respectively the equations

$$
\Delta_{y} \mu+\operatorname{div}_{y}\left[D_{p} H\left(D_{x} \bar{u}+D_{y} v, y\right) \mu\right]=0,
$$

and

$$
\begin{aligned}
& \bar{m}\left(\Delta_{y} \nu+\operatorname{div}_{y}\left[D_{p} H\left(D_{x} \bar{u}+D_{y} v, y\right) \nu\right]\right) \\
& =\mu \partial_{t} \bar{m}+\operatorname{div}_{x}\left[D_{p} H\left(D_{x} \bar{u}+D_{y} v, y\right) \mu\right]+2\left(D_{x} \bar{m}, D_{y} \mu\right) .
\end{aligned}
$$

Rewriting (3.2) with $p \in \mathbb{R}^{d}$ in place of $D_{x} \bar{u}(x, t)$ and $\bar{m} \in \mathbb{R}$ in place of $\bar{m}(x, t)$ we find

$$
\Delta_{y} \mu+\operatorname{div}_{y}\left[D_{p} H\left(D_{x} \bar{u}+D_{y} v, y\right) \mu\right]=0,
$$

which is the second equation of (4.9). 
The existence of $\nu$ in (3.3) follows from Fredholm's alternative provided that the integral of the right hand side of (3.3) over $\mathbb{T}^{d}$ vanishes. This requirement together with the normalization $\int_{\mathbb{T}^{d}} \mu(y) d y=1$, give the transport equation of the homogenized system, namely

$$
\partial_{t} \bar{m}+\operatorname{div}_{x}\left[\int_{\mathbb{T}^{d}}\left[D_{p} H\left(D \bar{u}+D_{y} v\right) \mu\right] d y \bar{m}\right]=0 .
$$

We continue with the nonlocal setting, which, in view of the assumption that the forcing term $F_{\epsilon}$ is independent of $x / \epsilon$, decouples. Indeed, the classical periodic homogenization theory, implies that, in view of (2.1) and (2.2), for each $p \in \mathbb{R}^{d}$, there exists a unique $\bar{H}(p)$ such that

$$
-\Delta v+H(D v+p, y)=\bar{H}(p)
$$

has a unique up to constants periodic solution.

It then follows that, for every $p \in \mathbb{R}^{d}, x \in \mathbb{R}^{d}$ and $m \in \mathcal{P}$, there exists a unique constant $\bar{H}(p, m, x)=$ $\bar{H}(p)-F(x, m)$ and a unique pair $(v, \mu) \in C^{2}\left(\mathbb{R}^{d}\right) \times L^{1}\left(\mathbb{T}^{d}\right)$, which are $\mathbb{T}^{d}$-periodic and

$$
\left\{\begin{array}{l}
-\Delta v+H(D v+p, y)-F(x, m)=\bar{H}(p, m, x) \text { in } \mathbb{T}^{d}, \\
\Delta \mu+\operatorname{div}[D H(D v+p, y) \mu]=0 \text { in } \mathbb{T}^{d}, \\
\int_{\mathbb{T}^{d}} v(y) d y=0, \mu>0 \text { in } \mathbb{T}^{d} \text { and } \int_{\mathbb{T}^{d}} \mu(y) d y=1 .
\end{array}\right.
$$

The local cell-problem

$$
\left\{\begin{array}{l}
-\Delta v+H(D v+p, y)-F(y, m \mu)=\bar{H}(p, m) \text { in } \mathbb{T}^{d}, \\
\Delta \mu+\operatorname{div}[D H(D v+p, y) \mu]=0 \text { in } \mathbb{T}^{d}, \\
\int_{\mathbb{T}^{d}} v(y) d y=0, \mu>0 \text { in } \mathbb{T}^{d} \text { and } \int_{\mathbb{T}^{d}} \mu(y) d y=1,
\end{array}\right.
$$

is a reparametrization of systems that have already solved in the literature, see, for example, [23] and [24], where we refer to for the details.

\section{Homogenization FOR MFG-SYSTEMS WITH NONLOCAL COUPLING}

In addition to the conditions on $H$ in section 2, here we assume that

$$
\text { there exist constants } C>c>0 \text { such that } c \leq \int_{\mathbb{R}^{d}} m_{T}(y) d y \leq C,
$$

and

$$
u_{0} \text { is Lipschitz continuous. }
$$

It follows from (4.1) and (4.2) and the assumptions on $H$ and $F$ that, for some constants $C, c>0$,

$$
\sup _{t \in[0, T]}\left\|D u^{\epsilon}(\cdot, t)\right\| \leq C \text { and } c \leq \inf _{t \in[0, T]} \int_{\mathbb{R}^{d}} m^{\epsilon}(x, t) d x \leq \sup _{t \in[0, T]} \int_{\mathbb{R}^{d}} m^{\epsilon}(x, t) d x \leq C .
$$

Since the bounds on $\int_{\mathbb{R}^{d}} m^{\epsilon}(x, t) d x$ are classical, here we only discuss the Lipschitz estimate on $u^{\epsilon}$. In view of (2.8), (2.9) and (2.10), such bounds follow from a Bernstein-type argument, see, for example, Lions and Souganidis [27], provided it is shown that there exists $C>0$ such that, for all sufficiently small $\epsilon>0$,

$$
\left\|\partial_{t} u^{\epsilon}\right\|_{\infty} \leq C
$$

Since it is immediate from that first equation of (1.1) that, for all $t \in[0, T]$,

$$
\left\|\partial_{t} u^{\epsilon}(\cdot, t)\right\|_{\infty} \leq\left\|\partial_{t} u^{\epsilon}(\cdot, T)\right\|_{\infty}+\int_{0}^{T}\left\|\partial_{s} F_{\epsilon}\left[m^{\epsilon}\right]\right\|_{\infty} d s \leq\left\|\partial_{t} u^{\epsilon}(\cdot, T)\right\|_{\infty}+\int_{0}^{T}\left\|<F_{\epsilon}^{\prime}\left[m^{\epsilon}\right], \partial_{s} m^{\epsilon}>\right\|_{\infty} d s,
$$

it is enough to obtain a bound for the quantity in the last integral above. 
This is where the regularity assumptions (2.8) and (2.9). Indeed differentiating the second equation in (1.1) in time and using (2.8) and (2.9) we find that $\left\|<F_{\epsilon}^{\prime}\left[m^{\epsilon}\right], \partial_{s} m^{\epsilon}>\right\|_{\infty}$ is controlled by $\left\|D_{p} H\left(D u^{\epsilon}, \cdot\right)\right\|_{\infty}$, which, in view of $(2.3)$ is bounded.

We state next the homogenization result, which also implies that the limiting system is of mfg-type.

Theorem 4.1. Assume that (1.1) is nonlocal, that is, (2.4), as well as (2.1), (2.2), (2.3), (2.5), (2.6), (2.8), (2.9), (2.10), (4.1). and (4.2). Then there exists a Hamiltonian $\bar{H}=H(p, m): \mathbb{R}^{d} \times \mathcal{P} \rightarrow \mathbb{R}$, which satisfies (2.2), (2.3), (2.6) and (4.1), such that, as $\epsilon \rightarrow 0, u^{\epsilon} \rightarrow \bar{u}$ locally uniformly in $\mathbb{R}^{d}$ and $m^{\epsilon} \rightarrow \bar{m}$ in $L_{t}^{\infty}\left(L_{x}^{1}\left(\mathbb{R}^{d}\right)\right)$, where $(\bar{u}, \bar{m})$ is the unique solution (1.10) with $\bar{b}$ satisfying (1.12).

Recall that $(\bar{u}, \bar{m})$ solves (1.10) if $\bar{u}$ is a viscosity solution of the Hamilton-Jacobi equation and $\bar{m}$ a distributional solution of the transport equation in in (1.10).

We continue with the proof.

The proof of Theorem 4.1. The arguments are based on a combination of the viscosity-type homogenization theory for the Hamilton-Jacobi-Bellman equation in (1.1) (see [26]) and the double scale limit-method for the transport equation in (1.1) (see [3], [28] and [13]). An additional argument is needed in the end to connect the two steps by showing the particular form of the limiting transport equation.

Throughout the proof all the limits $\epsilon \rightarrow 0$ will be taken along subsequences, a fact which will not be repeated. In addition, to keep the formulae shorter we often omit the explicit dependence of $u^{\epsilon}, D u^{\epsilon}$ and $m^{\epsilon}$ on $(x, t)$.

We begin with the two-scale convergence argument. In view of (4.3) and the assumptions on $H$, it follows that the families $\left(m^{\epsilon}\right)_{\epsilon>0}$ and $\left(D_{p} H\left(D u^{\epsilon}, \frac{\dot{-}}{\epsilon}\right) m^{\epsilon}\right)_{\epsilon>0}$ is are equibounded n $L^{1}\left(\mathbb{R}^{d} \times[0, T]\right)$.

Moreover, a simple calculation also yields that $\left(m^{\epsilon}\right)_{\epsilon>0}$ is weakly (in time) equicontinuous, that is, for all $\phi \in C_{c}\left(\mathbb{R}^{d}\right)$, the map $t \rightarrow \int_{\mathbb{R}^{d}} m^{\epsilon}(x, t) \phi(x) d x$ is continuous uniformly on $\epsilon$.

The two-scale convergence theory yields that, along subsequences, $\left(m^{\epsilon}\right)_{\epsilon>0}$ and $\left(D_{p} H\left(D u^{\epsilon}, \frac{\dot{-}}{\epsilon}\right) m^{\epsilon}\right)_{\epsilon>0}$ "double scale" converge respectively to $M: \mathbb{R}^{d} \times \mathbb{R}^{d} \times[0, \infty) \rightarrow \mathbb{R}$ and $\widetilde{M}=\left(\widetilde{M}_{1}, \ldots, \widetilde{M}_{d}\right): \mathbb{R}^{d} \times \mathbb{R}^{d} \times[0, \infty) \rightarrow \mathbb{R}^{d}$.

It follows that, as $\epsilon \rightarrow 0$, for every interval $I \subset(0, \infty)$, every smooth and $\mathbb{T}^{d}$-periodic with respect to its second argument $\phi: \mathbb{R}^{d} \times \mathbb{R}^{d} \times[0, T] \rightarrow \mathbb{R}$ and for $i=1, \ldots, d$,

$$
\begin{gathered}
\int_{0}^{T} \int_{\mathbb{R}^{d}} m^{\epsilon}(x, t) \phi\left(x, \frac{x}{\epsilon}, t\right) d x d t \rightarrow \int_{0}^{T} \int_{\mathbb{R}^{d}} \int_{\mathbb{T}^{d}} M(x, t) \phi(x, y, t) d x d y d t \\
\int_{0}^{T} \int_{\mathbb{R}^{d}} H_{p_{i}}\left(D u^{\epsilon}, \frac{x}{\epsilon}\right) m^{\epsilon} \phi\left(x, \frac{x}{\epsilon}, t\right) d x d t \rightarrow \int_{0}^{T} \int_{\mathbb{R}^{d}} \int_{\mathbb{T}^{d}} \widetilde{M}_{i}(x, t) \phi(x, y, t) d x d y d t,
\end{gathered}
$$

and, moreover,

$$
m^{\epsilon} \underset{\epsilon \rightarrow 0}{\rightarrow} \bar{m}=\bar{m}(x, t)=\int_{\mathbb{T}^{d}} M(x, y, t) d y \text { in } L^{1}\left(\mathbb{R}^{d} \times(0, T)\right) .
$$

In view of (4.2), (2.7) and (4.7) standard arguments from the theory of periodic homogenization of HamiltonJacobi equations (see, for example, Lions, Papanicolaou and Varadhan [26]), yield that, along subsequences, the family $\left(u^{\epsilon}\right)_{\epsilon>0}$ converges locally uniformly to a solution of

$$
\partial_{t} \bar{u}+\bar{H}(D \bar{u})-F(x, \bar{m})=0 \text { in } \mathbb{R}^{d} \times(0, T] \text { and } \bar{u}(\cdot, 0)=u_{0},
$$

where, for each $(p, m) \in \mathbb{R}^{d} \times L^{1}\left(\mathbb{R}^{d} \times(0, T)\right), \bar{H}=\bar{H}(p, m)$ is the effective Hamiltonian, that is, unique constant such that the cell problem

$$
-\Delta w+H(D w+p, y)=\bar{H}(p) \text { in } \mathbb{T}^{d}
$$

has a periodic smooth solution, often referred to as the corrector, which is unique if it is normalized by

$$
\int_{\mathbb{T}^{d}} w(y) d y=0
$$


Multiplying the transport equation in (1.1) by $\phi(x)+\epsilon v\left(x, \frac{x}{\epsilon}\right)$, where $\phi \in C_{c}^{2}\left(\mathbb{R}^{d}\right)$ and $v \in C_{c, p}^{2}\left(\mathbb{R}^{d} \times \mathbb{T}^{d}\right)$, and integrating over $\mathbb{R}^{d}$ we find

$$
\begin{array}{r}
\left.\frac{d}{d t} \int_{R^{d}} m^{\epsilon}\left[\phi(x)+\epsilon v\left(x, \frac{x}{\epsilon}\right)\right] d x-\int_{R^{d}}\left(D_{p} H\left(D u^{\epsilon}, \frac{x}{\epsilon}\right) m^{\epsilon}, D \phi(x)+\left[\epsilon D_{x}+D_{y}\right] v\left(x, \frac{x}{\epsilon}\right)\right]\right) d x \\
=-\int_{R^{d}} m^{\epsilon}\left(\epsilon \Delta \phi(x)+\left[\epsilon^{2} \Delta_{x}+2 \Delta_{x y}+\Delta_{y}\right] v\left(x, \frac{x}{\epsilon}\right)\right) d x
\end{array}
$$

Letting $\phi \equiv 0$ in (4.11) and using (4.6) yields, after letting $\epsilon \rightarrow 0$ and integrating over $[0, T]$, that, for all $v \in C_{c, p}^{2}\left(\mathbb{R}^{d} \times \mathbb{T}^{d}\right)$,

$$
\int_{0}^{T} \int_{\mathbb{R}^{d}} \int_{\mathbb{T}^{d}}\left[\left(D_{y} v, \widetilde{M}\right)-\Delta_{y} v M\right] d x d y d t=0 .
$$

Similarly, if $v \equiv 0$ in (4.11), letting $\epsilon \rightarrow 0$ and using (4.7), we get

$$
\partial_{t} m+\operatorname{div} \int_{\mathbb{T}^{d}} \widetilde{M}(x, y, t) d y=0 .
$$

To conclude we need to show that

$$
\int_{\mathbb{T}^{d}} \widetilde{M}(x, y, t) d y=D_{p} \bar{H}(D \bar{u}) \int_{\mathbb{T}^{d}} M(x, y, t) d y=D_{p} \bar{H}(D \bar{u}) \bar{m} .
$$

We combine next (4.8) and (4.13) in the usual way, that is, we multiply (4.8) by $M$ and (4.13) by $u$ and integrate in $x$ and $y$. It follows that

$$
\frac{d}{d t} \int_{\mathbb{R}^{d}} \int_{\mathbb{T}^{d}} \bar{u}(x, t) M(x, y, t) d y d x+\int_{\mathbb{R}^{d}} \int_{\mathbb{T}^{d}}[\bar{H}(D \bar{u}) M-F(x, \bar{m}) M-(D \bar{u}, \widetilde{M})] d y d x=0 .
$$

A similar caIculation at the $\epsilon$-level, which also classical in the mfg-theory yields, for each $\epsilon>0$,

$$
\left.\frac{d}{d t} \int_{\mathbb{R}^{d}} u^{\epsilon} m^{\epsilon} d x+\int_{\mathbb{R}^{d}}\left[H\left(D u^{\epsilon}, \frac{x}{\epsilon}\right)-F\left(x, m^{\epsilon}\right)-\left(D u^{\epsilon}, D_{p} H\left(D u^{\epsilon}, \frac{x}{\epsilon}\right)\right)\right] m^{\epsilon}\right] d x=0 .
$$

The local uniform and weak convergence of $u^{\epsilon}$ and $m^{\epsilon}$ respectively give that, as $\epsilon \rightarrow 0$,

$$
\int_{0}^{T} \int_{\mathbb{R}^{d}} u^{\epsilon} m^{\epsilon} d x d t \rightarrow \int_{0}^{T} \int_{\mathbb{R}^{d}} \int_{\mathbb{T}^{d}} \bar{u} M d y d x d t
$$

Combining this last fact with (4.15) and (4.16) we find, again as $\epsilon \rightarrow 0$,

$$
\begin{array}{r}
\int_{0}^{T} \int_{\mathbb{R}^{d}}\left[\left(H\left(D u^{\epsilon}, \frac{x}{\epsilon}\right)-F\left(x, m^{\epsilon}\right)\right) m^{\epsilon}-\left(D u^{\epsilon}, D_{p} H\left(D u^{\epsilon}, \frac{x}{\epsilon}\right) m^{\epsilon}\right)\right] d x d t \rightarrow \\
\int_{0}^{T} \int_{\mathbb{R}^{d}} \int_{\mathbb{T}^{d}}[(\bar{H}(D \bar{u})-F(x, \bar{m})) M-D \bar{u} \cdot \widetilde{M}] d y d x .
\end{array}
$$

It also follows from the definition of the two-scale limit that, as $\epsilon \rightarrow 0$,

$$
\int_{0}^{T} \int_{\mathbb{R}^{d}} H\left(D \bar{u}+D w\left(\frac{x}{\epsilon}\right), \frac{x}{\epsilon}\right) m^{\epsilon} d x d t \rightarrow \int_{0}^{T} \int_{\mathbb{R}^{d}} \int_{\mathbb{T}^{d}} H(D \bar{u}+D w) M d y d x d t,
$$

and

$$
\int_{0}^{T} \int_{\mathbb{R}^{d}}\left(D_{p} H\left(D \bar{u}+D w\left(\frac{x}{\epsilon}\right), \frac{x}{\epsilon}\right) m^{\epsilon},\left(D \bar{u}+D w\left(\frac{x}{\epsilon}\right)\right)\right) \rightarrow \int_{0}^{T} \int_{\mathbb{R}^{d}} \int_{\mathbb{T}^{d}}(\widetilde{M},(D \bar{u}+D w)) d y d x d t .
$$

Combining all the previous facts we find that, as $\epsilon \rightarrow 0$,

$$
\begin{aligned}
\int_{0}^{T} \int_{\mathbb{R}^{d}}[H(D \bar{u} & \left.+D w\left(\frac{x}{\epsilon}\right), \frac{x}{\epsilon}\right)-H\left(D u^{\epsilon}, \frac{x}{\epsilon}\right) \\
& \left.-\left(D_{p} H\left(D u^{\epsilon}, \frac{x}{\epsilon}\right), D \bar{u}+D w\left(\frac{x}{\epsilon}\right)-D u^{\epsilon}\right)\right] m^{\epsilon} d x d t \rightarrow 0 .
\end{aligned}
$$


The uniform convexity of $H$ and (4.20) then yield that, as $\epsilon \rightarrow 0$,

$$
\int_{0}^{T} \int_{\mathbb{R}^{d}}\left|D \bar{u}+D w\left(\frac{x}{\epsilon}\right)-D u^{\epsilon}\right|^{2} m^{\epsilon} d x d t \rightarrow 0,
$$

and, hence, as $\epsilon \rightarrow 0$,

$$
\int_{0}^{T} \int_{\mathbb{R}^{d}}\left|D \bar{u}+D w\left(\frac{x}{\epsilon}\right)-D u^{\epsilon}\right| m^{\epsilon} d x d t \rightarrow 0 .
$$

The last claim follows from the observation that, if, for some $C>0,\left|G^{\epsilon}\right| \leq C$ in $\mathbb{R}^{d} \times[0, T]$ and, as $\epsilon \rightarrow 0$,

$$
\int_{0}^{T} \int_{\mathbb{R}^{d}}\left|G^{\epsilon}(x, t)\right|^{2} m^{\epsilon}(x, t) d x d t \rightarrow 0
$$

then

$$
\begin{aligned}
\int_{0}^{T} \int_{\mathbb{R}^{d}}\left|G^{\epsilon}(x, t)\right| m^{\epsilon}(x, t) d x d t & \leq \int_{0}^{T}\left[\int_{\mathbb{R}^{d}}\left|G^{\epsilon}(x, t)\right|^{2} m^{\epsilon}(x, t) d x\right]^{1 / 2}\left[\int_{\mathbb{R}^{d}} m^{\epsilon}(x, t) d x\right]^{1 / 2} d t \\
& \leq \int_{0}^{T}\left[\int_{\mathbb{R}^{d}}\left|G^{\epsilon}(x, t)\right|^{2} m^{\epsilon}(x, t) d x\right]^{1 / 2} d t \underset{\epsilon \rightarrow 0}{\rightarrow} 0 .
\end{aligned}
$$

The Lipschitz continuity of $D H$ and (4.22) then give that, as $\epsilon \rightarrow 0$, for all $\phi \in C_{c, p}^{2}\left(\mathbb{R}^{d} \times \mathbb{T}^{d}\right)$ and $i=1, \ldots, d$,

$$
\int_{0}^{T} \int_{\mathbb{R}^{d}}\left[H_{p_{i}}\left(D u^{\epsilon}, \frac{x}{\epsilon}\right)-H_{p_{i}}\left(D \bar{u}+D w\left(\frac{x}{\epsilon}\right), \frac{x}{\epsilon}\right)\right] m^{\epsilon} \phi\left(x, \frac{x}{\epsilon}\right) d x d t \rightarrow 0 .
$$

Using $H_{p_{i}}(D \bar{u}+D w(y), y) \phi(x, y, t)$ as test function in the double-scale limit of $m^{\epsilon}$ we find that, as $\epsilon \rightarrow 0$, for all $\phi \in C_{c, p}\left(\mathbb{R}^{d} \times \mathbb{T}^{d}\right)$ and $i=1, \ldots, d$,

$$
\int_{0}^{T} \int_{\mathbb{R}^{d}}\left[H_{p_{i}}\left(D u^{\epsilon}, \frac{x}{\epsilon}\right) m^{\epsilon} \phi\left(x, \frac{x}{\epsilon}, t\right) d x d t \rightarrow \int_{0}^{T} \int_{\mathbb{R}^{d}} \int_{\mathbb{T}^{d}} H_{p_{i}}\left(D \bar{u}+D_{y} w, y\right) M(x, y, t) \phi(x, y, t) d x d y d t,\right.
$$

and, hence, in view of (4.6),

$$
\widetilde{M}=D_{p} H(D \bar{u}+D w, y) M
$$

It follows then follows from (4.13) and (4.23) that

$$
\partial_{t} \bar{m}+\operatorname{div}_{x}\left(\int_{\mathbb{T}^{d}} D_{p} H(D \bar{u}+D w, y) M(x, y, t) d y\right)=0 \text { in } \mathbb{R}^{d} \times[0, T) .
$$

It remains to show that

$$
\int_{\mathbb{T}^{d}} D_{p} H(D \bar{u}+D w, y) M(x, y, t) d y=\bar{H}_{p_{i}}(D \bar{u}) \bar{m} .
$$

Differentiating (4.9) with respect to $p$ we find, for each $i=1, \ldots, d$,

$$
-\Delta w_{p_{i}}+D_{p} H\left(p+D_{y} w, y\right) \cdot D w_{p_{i}}+H_{p_{i}}=\bar{H}_{p_{i}} \text { in } \mathbb{R}^{d} .
$$

The argument above can be justified by taking difference quotients in (4.9) and passing in the limiting using the continuity properties of the ergodic constant. We leave the details to the reader.

Let $p=D \bar{u}(x, t)$ in (4.27), multiply by $\phi M$ with $\phi \in C_{c}\left(\mathbb{R}^{d} \times[0, T]\right)$, integrate over $\mathbb{R}^{d} \times \mathbb{T}^{d} \times[0, T]$ and use (4.12) to get

$$
\int_{0}^{T} \int_{\mathbb{R}^{d}} \int_{\mathbb{T}^{d}} \phi(x) H_{p_{i}}\left(D_{x} \bar{u}+D_{y} w, y\right) M d y d x d t=\int_{0}^{T} \int_{\mathbb{R}^{d}} \int_{\mathbb{T}^{d}} \bar{H}_{p_{i}}(D \bar{u}) M d y d x d t,
$$

hence, (4.26) and (4.14) hold, and, thus

$$
\partial_{t} \bar{m}+\operatorname{div}\left(D_{p} \bar{H}(D \bar{u}) \bar{m}\right)=0 \text { in } \mathbb{R}^{d} \times[0, T) .
$$


We turn next to the well-posedness.

The proof of Theorem ??. The fact that $F$ is independent of $y$ and the uniqueness of the ergodic constant $\bar{H}$ yield that, for each $p \in \mathbb{R}^{d}$ and $m \in L^{1}\left(\mathbb{R}^{d}\right)$,

$$
\bar{H}(p, m)=\bar{h}(p)-F(m)
$$

where $\bar{h}(p)$ is the unique constant for which the cell problem

$$
-\Delta_{y} w+H\left(p+D_{y} w, y\right)=\bar{h}(p) \text { in } \mathbb{R}^{d}
$$

has a $\mathbb{T}^{d}$-periodic solution.

It is well known, see, for example, [26], that the convexity of $H$ implies the convexity of $\bar{h}$. Since $F$ is the same as in (1.1), $\bar{H}$ has the properties needed for the limit mfg-system to have a unique solution.

\section{HomogeniZATION FOR MFG-SYSTEMS WITH LOCAL COUPLING}

When dealing with (1.1) with local coupling we loose the regularizing property which allowed in the previous section to pass the weak limit of the $m^{\epsilon}$ 's in the nonlinearity, which, in turn, essentially decoupled the cellproblem system.

Instead of introducing conditions that lead in some cases to independent of $\epsilon$ apriori bounds on $\left(u^{\epsilon}, m^{\epsilon}\right)$, here we concentrate on proving a homogenization result for (1.1) assuming that the limit system has a classical solution, which is true when the horizon $T$ using is small.

We work with well-prepared initial and terminal conditions, that is, we assume that

$$
u_{0}^{\epsilon}(x)=\bar{u}_{0}(x)+\epsilon v\left(\frac{x}{\epsilon}\right) \text { and } m_{T}^{\epsilon}(x)=\bar{m}_{T}(x)\left(\mu\left(\frac{x}{\epsilon}\right)+\epsilon \nu\left(\frac{x}{\epsilon}\right),\right.
$$

where $\bar{u}_{0}$ and $\bar{m}_{T}$ satisfy respectively (4.2) and (4.1) and $v, \mu$ and $\nu$ are constructed from $\bar{u}_{0}$ and $\bar{m}_{T}$ as in the course fo the proof of Theorem 5.1.

Theorem 5.1. Assume that (1.1) is local, that is, (1.5) and (1.6), and, in addition, (2.1), (2.2), (2.3), (2.16), either (2.17) or (??) and fix $\bar{u}_{0} \in C_{b}^{2}\left(\mathbb{R}^{d}\right) \cap H^{1}\left(\mathbb{R}^{d}\right)$ and $\bar{m}_{T} \in C_{b}^{2}\left(\mathbb{R}^{d}\right) \cap L^{1}\left(\mathbb{R}^{d}\right)$ satisfying respectively (4.2) and (4.1). Moreover, assume that the system (1.10) with initial and terminal condition $\bar{u}_{0}$ and $\bar{m}_{T}$ respectively and $\bar{H}$ as in (4.10) and $\bar{b}$ given by (1.11) has a classical solution $(\bar{u}, \bar{m})$. Finally, let $\left(u^{\epsilon}, m^{\epsilon}\right)$ be the solution of (1.1) with initial and terminal condition $\bar{u}_{0}^{\epsilon}$ and $\bar{m}_{T}^{\epsilon}$ as in (5.1). Then, as $\epsilon \rightarrow 0, u^{\epsilon} \rightarrow \bar{u}$ in $H^{1}\left(\mathbb{R}^{d} \times(0, T)\right)$ and $m^{\epsilon} \rightarrow \bar{m}$ in $L^{1}\left(\mathbb{R}^{d}\right)$.

Proof. The proof is based on constructing, using the regularity of $(\bar{u}, \bar{m})$ and the ansatz (1.7), an approximate solution of (1.1), which is very close to $\left(u^{\epsilon}, m^{\epsilon}\right)$.

The assumption that on the initial and terminal conditions of (1.1) allows for a significant simplification of the proof, which, nevertheless is tedious.

We assume that the solution $(v(\cdot ; p, m), \mu(\cdot ; p, m))$ of $(4.9)$ depends smoothly on $(p, m)$, introduce the functions $\tilde{v}$ and $\tilde{\mu}$ given by

$$
\tilde{v}(y, x, t)=v(y ; D \bar{u}(x, t), \bar{m}(x, t)) \text { and } \tilde{\mu}(x, y, t)=\mu(y ; D \bar{u}(x, t), \bar{m}(x, t)),
$$

and claim that there exists a smooth $\tilde{\nu}: \mathbb{T}^{d} \times \mathbb{R}^{d} \times[0, T] \rightarrow \mathbb{R}$ such that

$$
\widehat{u}^{\epsilon}(x, t)=\bar{u}(x, t)+\epsilon \tilde{v}\left(\frac{x}{\epsilon}, x, t\right) \text { and } \widehat{m}^{\epsilon}(x, t)=\bar{m}(x, t)\left(\tilde{\mu}\left(\frac{x}{\epsilon}, x, t\right)+\tilde{\nu}\left(\frac{x}{\epsilon}, x, t\right)\right)
$$

is a solution of (1.1) up to an error, which is small in a sense that will become clear in the course.

Notice that

$$
u_{0}(x)=\widehat{u}^{\epsilon}(x, 0) \text { and } m_{T}(x)=\widehat{m}^{\epsilon}(x, T) .
$$

We begin with the first equation in (1.1). In what follows, we use that

$$
\partial_{t} \bar{u}+\bar{H}(D \bar{u}, \bar{m})=0 \text { and }-\Delta_{y} v+H\left(D \bar{u}+D_{y} v, y 0-F(y, \bar{m} \mu)=\bar{H}(D \bar{u}, \bar{m})\right.
$$

and omit, whenever it does not create confusion, the dependence of all functions on their arguments. 
Inserting the formula for $\widehat{u}^{\epsilon}$ in the Bellman equation of (1.1) we find

$$
\begin{aligned}
& \partial_{t} \widehat{u}^{\epsilon}-\epsilon \Delta \widehat{u}^{\epsilon}+H\left(D \widehat{u}^{\epsilon}, \frac{x}{\epsilon}\right)-F\left(\frac{x}{\epsilon}, \widehat{m}^{\epsilon}\right)=\partial_{t} \bar{u}+\epsilon \partial_{t} \tilde{v}-\epsilon \Delta \bar{u}-\left(\Delta_{y} \tilde{v}+2 \epsilon \Delta_{x, y} \tilde{v}+\epsilon^{2} \Delta_{x} \tilde{v}\right) \\
& +H\left(D \bar{u}+D_{y} \tilde{v}+\epsilon D_{x} \tilde{v}, \frac{x}{\epsilon}\right)-F\left(\frac{x}{\epsilon}, \bar{m}(\tilde{\mu}+\epsilon \tilde{\nu})\right)=\partial_{t} \bar{u}+\bar{H}(D \bar{u}, \bar{m}) \\
& -\Delta_{y} v\left(\frac{x}{\epsilon} ; D \bar{u}, \bar{m}\right)+H\left(D \bar{u}+D_{y} v\left(\frac{x}{\epsilon} ; D \bar{u}, \bar{m}\right), \frac{x}{\epsilon}\right)-F\left(\frac{x}{\epsilon}, \bar{m} \mu\left(\frac{x}{\epsilon} ; D \bar{u}, \bar{m}(x, t)\right)-\bar{H}(D \bar{u}, \bar{m})=A^{\epsilon}(x, t),\right.
\end{aligned}
$$

where

$$
\begin{aligned}
& A^{\epsilon}(x, t)=\epsilon \partial_{t} \tilde{v}-\epsilon \Delta \bar{u}-2 \epsilon \Delta_{x, y} \tilde{v}-\epsilon^{2} \Delta_{x} \tilde{v}+H\left(D \bar{u}+D_{y} v+\epsilon D_{x} \tilde{v}, \frac{x}{\epsilon}\right) \\
& \left.-F\left(\frac{x}{\epsilon}, \bar{m}(\tilde{\mu}+\epsilon \tilde{\nu})\left(\frac{x}{\epsilon}, x, t\right)\right)-H\left(D \bar{u}+D_{y} v, \frac{x}{\epsilon}\right)+F\left(\frac{x}{\epsilon}, \bar{m} \mu\right)\right)
\end{aligned}
$$

note that above we used the fact that $D_{y} \tilde{v}=D_{y} v$.

The assumptions on $H$ and $F$ and the regularity of $\bar{u}$ and $\bar{m}$ yield $A_{1}^{\epsilon} \in C_{b}\left(\mathbb{R}^{d} \times \mathbb{R} ; \mathbb{R}^{d}\right)$ and $A_{2}^{\epsilon} \in C_{b}\left(\mathbb{R}^{d} \times \mathbb{R} ; \mathbb{R}^{\prime}\right.$ such that

$$
\begin{aligned}
& H\left(D \bar{u}+\left(D_{y}+\epsilon D_{x}\right) \tilde{v}, \frac{x}{\epsilon}\right)-F\left(\frac{x}{\epsilon},(\tilde{\mu}+\epsilon \tilde{\nu})\left(\frac{x}{\epsilon}, x, t\right)\right)= \\
& H\left(D \bar{u}+D_{y} v\left(\frac{x}{\epsilon} ; D \bar{u}, \bar{m}\right), \frac{x}{\epsilon}\right)-F\left(\frac{x}{\epsilon}, \bar{m} \mu\left(\frac{x}{\epsilon} ; D \bar{u}, \bar{m}\right)\right)+\epsilon\left(A_{1}^{\epsilon}, D_{x} \tilde{v}\right)+\epsilon A_{2}^{\epsilon} \tilde{\nu}
\end{aligned}
$$

and, hence, for some $C>0$,

$$
\left|A^{\epsilon}\right| \leq C \text { on } \mathbb{R}^{d} \times[0, T]
$$

that is,

$$
\left\|\partial_{t} \widehat{u}^{\epsilon}-\epsilon \Delta \widehat{u}^{\epsilon}+H\left(D \widehat{u}^{\epsilon}, \frac{x}{\epsilon}\right)-F\left(\frac{x}{\epsilon}, \widehat{m}^{\epsilon}\right)\right\|_{L^{\infty}\left(\mathbb{R}^{d} \times[0, T]\right)} \leq C \epsilon .
$$

Justifying the above is a long but nevertheless routine calculus exercise, which requires knowing that $\tilde{\nu}$ as well as $D_{p} v, D_{m} v, D_{p} \mu$ and $D_{m} \mu$ are uniformly bounded. The existence of such $\tilde{\nu}$ is discussed below. The other bounds follow from differentiating (4.9) with respect to $p$ and $m$ and studying the resulting problems. We leave these details up to the reader.

The argument for the second equation of (1.1) is slightly more complicated since it involves the two scale convergence. To simplify the notation, we find it necessary to have some preliminary discussion and to introduce some useful notation. As before, when it does not create confusion, we omit the explicit dependence on the variables.

We begin with the observation that the independent of $\epsilon$ bounds on $D_{x} \tilde{v}$ and $D_{x} \tilde{\mu}$ imply that there exist $b_{1}^{\epsilon}, b_{2}^{\epsilon} \in C_{b}^{1}\left(\mathbb{R}^{d} \times[0, T] ; \mathbb{R}^{d}\right)$ with $C^{1}$-bounds independent of $\epsilon$ such that

$$
D_{p} H\left(D \widehat{u}^{\epsilon}, \frac{x}{\epsilon}\right)=D_{p} H\left(D_{x} \bar{u}+D_{y} v+\epsilon D_{x} \tilde{v}, \frac{x}{\epsilon}\right)=D_{p} H\left(D \bar{u}+D_{y} v, \frac{x}{\epsilon}\right)+\epsilon b_{1}^{\epsilon}+\epsilon^{2} b_{2}^{\epsilon}
$$


We return now to the second equation of (1.1). In what follows, to simplify the notation we write $\mu$ and $\nu$ in place of $\tilde{\mu}$ and $\tilde{\nu}$. We find that

$$
\begin{aligned}
& \partial_{t} \widehat{m}^{\epsilon}+\epsilon \Delta \widehat{m}^{\epsilon}+\operatorname{div}\left[D_{p} H\left(D \widehat{u}^{\epsilon}\right) \widehat{m}^{\epsilon}\right]= \\
& \partial_{t} \bar{m}(\mu+\epsilon \nu)+\bar{m} \partial_{t}(\mu+\epsilon \nu)+\epsilon \Delta_{x} \bar{m}(\mu+\epsilon \nu)+\bar{m}\left(\frac{1}{\epsilon} \Delta_{y}+2 \Delta_{x y}+\epsilon \Delta_{x}\right)(\mu+\epsilon \nu)+2\left(D_{x} \bar{m}, D_{y}(\mu+\epsilon n)\right) \\
& +2 \epsilon\left(D_{x} \bar{m}, D_{x}(\mu+\epsilon n)\right)+\frac{1}{\epsilon} \operatorname{div}_{y}\left[D_{p} H\left(D \bar{u}+D_{y} v, \frac{x}{\epsilon}\right) \bar{m} \mu\right]+\operatorname{div}_{x}\left[D_{p} H\left(D \bar{u}+D_{y} v, \frac{x}{\epsilon}\right) \bar{m} \mu\right] \\
& +\operatorname{div}_{y}\left[D_{p} H\left(D \bar{u}+D_{y} v, \frac{x}{\epsilon}\right) \bar{m} \nu\right]+\epsilon \operatorname{div}_{x}\left[D_{p} H\left(D \bar{u}+D_{y} v, \frac{x}{\epsilon}\right) \bar{m} \nu\right]+\operatorname{div}_{y}\left[b_{1}^{\epsilon} \bar{m} \mu\right] \\
& +\epsilon \operatorname{div}_{x}\left[b_{1}^{\epsilon} \bar{m} \mu\right]+\epsilon\left(\operatorname{div}_{y}\left[b_{1}^{\epsilon} \bar{m} \nu\right]+\epsilon \operatorname{div}_{x}\left[b_{1}^{\epsilon} \bar{m} \nu\right]\right)+\epsilon\left(\operatorname{div}_{y}\left[b_{2}^{\epsilon} \bar{m} \mu\right]+\epsilon \operatorname{div}_{x}\left[b_{2}^{\epsilon} \bar{m} \mu\right]\right) \\
& +\epsilon^{2}\left(\operatorname{div}_{y}\left[b_{2}^{\epsilon} \bar{m} \nu\right]+\epsilon \operatorname{div}_{x}\left[b_{2}^{\epsilon} \bar{m} \nu\right]\right) .
\end{aligned}
$$

Reorganizing the identities above yields

$$
\partial_{t} \widehat{m}^{\epsilon}+\epsilon \Delta \widehat{m}^{\epsilon}+\operatorname{div}\left[D_{p} H\left(D \widehat{u}^{\epsilon}\right) \widehat{m}^{\epsilon}\right]=\frac{1}{\epsilon} B_{1}^{\epsilon}+B_{2}^{\epsilon}+\epsilon B^{\epsilon},
$$

where

$$
\begin{gathered}
B_{1}^{\epsilon}=\bar{m}\left(\Delta_{y} \mu+\operatorname{div}_{y}\left[D_{p} H\left(D \bar{u}+D_{y} v, \frac{x}{\epsilon}\right) \mu\right]\right), \\
B_{2}^{\epsilon}=\bar{m}\left(\Delta_{y} \nu+\operatorname{div}_{y}\left[D_{p} H\left(D \bar{u}+D_{y} v, \frac{x}{\epsilon}\right) \nu\right]+\partial_{t} \mu+2 \Delta_{x y} \mu+2\left(D_{x} \bar{m}, D_{y} \mu\right)\right) \\
+\partial_{t} \bar{m} \mu+\operatorname{div}_{x}\left[D_{p} H\left(D \bar{u}+D_{y} v, \frac{x}{\epsilon}\right) \bar{m} \mu\right]+\operatorname{div}_{y}\left[b_{1}^{\epsilon} \bar{m} \mu\right],
\end{gathered}
$$

and

$$
\begin{aligned}
B_{3}^{\epsilon}= & \partial_{t} \bar{m} \nu+\bar{m} \partial_{t} \nu+\Delta \bar{m}(\mu+\epsilon \nu)+\bar{m} \Delta_{x}(\mu+\epsilon \nu)+2\left(D_{x} \bar{m}, D_{y} \nu\right)+2\left(D_{x} \bar{m}, D_{x}(\mu+\epsilon \nu)\right) \\
& +\operatorname{div}_{x}\left[D_{p} H\left(D \bar{u}+D_{y} v, \frac{x}{\epsilon}\right) \bar{m} \nu\right]+\operatorname{div}_{x}\left[b_{1}^{\epsilon} \bar{m} \mu\right]+\operatorname{div}_{y}\left[b_{1}^{\epsilon} \bar{m} \nu\right]+\operatorname{div}_{x}\left[b_{1}^{\epsilon} \bar{m} \nu\right] \\
& +\left(\operatorname{div}_{y}\left[b_{2}^{\epsilon} \bar{m} \mu\right]+\operatorname{div}_{x}\left[b_{2}^{\epsilon} \bar{m} \mu\right]\right)+\epsilon\left(\operatorname{div}_{y}\left[b_{2}^{\epsilon} \bar{m} \nu\right]+\epsilon \operatorname{div}_{x}\left[b_{2}^{\epsilon} \bar{m} \nu\right]\right) .
\end{aligned}
$$

The choice of $\mu$ implies that $B_{1}^{\epsilon}=0$. Moreover, using Fredholm's alternative it is also possible to find a $\mathbb{T}^{d}$-periodic $\nu$ such that $B_{2}^{\epsilon}=0$. Indeed, the choice of $\bar{m}$ and the fact that $\int_{\mathbb{T}^{d}} \mu=1$ imply that

$$
\int_{\mathbb{T}^{d}}\left(\partial_{t} \mu+2 \Delta_{x y} \mu+2\left(D_{x} \bar{m}, D_{y} \mu\right)+\frac{1}{\bar{m}}\left[\partial_{t} \bar{m} \mu+\operatorname{div}_{x}\left[D_{p} H\left(D \bar{u}+D_{y} v, \frac{x}{\epsilon}\right) \bar{m} \mu\right]+\operatorname{div}_{y}\left[b_{1}^{\epsilon} \bar{m} \mu\right]\right]\right)=0 .
$$

Finally, it is immediate from the assumed regularity of $\bar{m}$ that $B_{3}^{\epsilon} \mathrm{E}^{1}\left(\mathbb{R}^{d} \times[0, T]\right)$ with norm bounded independently of $\epsilon$, and, hence, there exists $C>0$ such that

$$
\left\|\partial_{t} \widehat{m}^{\epsilon}+\epsilon \Delta \widehat{m}^{\epsilon}+\operatorname{div}\left[D_{p} H\left(D \widehat{u}^{\epsilon}\right) \widehat{m}^{\epsilon}\right]\right\|_{L^{1}\left(\mathbb{R}^{d} \times[0, T]\right)} \leq C \epsilon .
$$

Next we compare $\left(u^{\epsilon}, m^{\epsilon}\right)$ to $\left(\widehat{u}^{\epsilon}, \widehat{m}^{\epsilon}\right)$ using the typical in the mfg-theory argument consisting of writing the equations for $u^{\epsilon}-\widehat{u}^{\epsilon}$ and $m^{\epsilon}-\widehat{m}^{\epsilon}$. It follows that

$$
\left\{\begin{aligned}
& \partial_{t}\left(u^{\epsilon}-\widehat{u}^{\epsilon}\right)-\epsilon \Delta\left(u^{\epsilon}-\widehat{u}^{\epsilon}\right) \\
&+H\left(D u^{\epsilon}, \frac{x}{\epsilon}\right)-F\left(\frac{x}{\epsilon}, m^{\epsilon}\right)-H\left(D \widehat{u}^{\epsilon}, \frac{x}{\epsilon}\right)+F\left(\frac{x}{\epsilon}, \widehat{m}^{\epsilon}\right)=\mathrm{O}_{L^{\infty}}(\epsilon) \text { in } \mathbb{R}^{d} \times(0, T], \\
& \partial_{t}\left(m^{\epsilon}-\widehat{m}^{\epsilon}\right)+\epsilon \Delta\left(m^{\epsilon}-\widehat{m}^{\epsilon}\right)+ \\
&\left.\operatorname{div}\left[D_{p} H\left(D u^{\epsilon}, \frac{x}{\epsilon}\right) m^{\varepsilon}\right)-D_{p} H\left(D \widehat{u}^{\epsilon}, \frac{x}{\epsilon}\right) \widehat{m}^{\epsilon}\right]=\mathrm{O}_{L^{1}}(\epsilon) \text { in } \mathbb{R}^{d} \times(0, T], \\
&\left(u^{\epsilon}-\widehat{u}^{\epsilon}\right)(\cdot, 0)=0 \text { and }\left(m^{\epsilon}-\widehat{m}^{\epsilon}\right)(\cdot, T)=0 .
\end{aligned}\right.
$$


Multiplying the first equation of (5.7) by $m^{\epsilon}-\widehat{m}^{\epsilon}$ and the second by $u^{\epsilon}-\widehat{u}^{\epsilon}$, integrating over $\mathbb{R}^{d} \times[0, T]$, adding the two integrals and using the initial and terminal conditions in (5.7) yields

$$
\begin{gathered}
\int_{0}^{T} \int_{\mathbb{R}^{d}}\left[\left(m^{\epsilon}-\widehat{m}^{\epsilon}\right)\left(H\left(D u^{\epsilon}, \frac{x}{\epsilon}\right)-F\left(\frac{x}{\epsilon}, m^{\epsilon}\right)-H\left(D \widehat{u}^{\epsilon}, \frac{x}{\epsilon}\right)+F\left(\frac{x}{\epsilon}, \widehat{m}^{\epsilon}\right)\right)\right. \\
\left.\left.-\left(D\left(u^{e} p-\widehat{u}^{\epsilon}\right), D_{p} H\left(D u^{\epsilon}, \frac{x}{\epsilon}\right) m^{\varepsilon}\right)-D_{p} H\left(D \widehat{u}^{\epsilon}, \frac{x}{\epsilon}\right) \widehat{m}^{\epsilon}\right)\right] d x d t=\mathrm{O}(\epsilon) .
\end{gathered}
$$

It follows from the uniform convexity of $H$, the monotonicity with respect to $m$ and the assumed strict positivity of $\bar{m}$, which yields a positive and independent of $\epsilon$ lower bound for $\widehat{m}^{\epsilon}$, that $\lim _{\epsilon \rightarrow 0}\left\|D\left(u^{\epsilon}-\widehat{u}^{\epsilon}\right)\right\|_{L^{2}}$ and, since $u^{\epsilon}-\widehat{u}^{\epsilon}(\cdot, 0)$, that $\lim _{\epsilon \rightarrow 0}\left\|u^{\epsilon}-\widehat{u}^{\epsilon}\right\|_{H^{1}}$, and, then, that $m^{\epsilon}-\widehat{m}^{\epsilon} \rightarrow 0$ in $L^{1}$.

We investigate next whether the limit system (1.10) is of mfg-type or not, that is, if (1.12) holds. The answer is, in general, negative at least when dealing with potential mfg as we explain next.

We consider the mfg

$$
\left\{\begin{array}{l}
\partial_{t} u^{\epsilon}+\epsilon \Delta u^{\epsilon}+H\left(D u^{\epsilon}, \frac{x}{\epsilon}\right)-F\left(\frac{x}{\epsilon}, m^{\epsilon}\right)=0 \text { in } \mathbb{R}^{d} \times[0, T), \\
\partial_{t} m^{\epsilon}-\epsilon \Delta m^{\epsilon}+\operatorname{div}\left[D H\left(D u^{\epsilon}, \frac{x}{\epsilon}\right) m^{\epsilon}\right] \text { in } \mathbb{R}^{d} \times(0, T], \\
u^{\epsilon}(\cdot, T)=u_{T}^{\epsilon} \text { and } m^{\epsilon}(\cdot, 0)=m_{0},
\end{array}\right.
$$

and assume that

$$
\text { there exists a } \mathbb{T}^{d} \text {-periodic and convex } \mathcal{F}: \mathbb{R}^{d} \times \mathbb{R} \text { such that } F(y, m)=\frac{\delta \mathcal{F}}{\delta m}(y, m ;
$$

notice that to be consistent with the classical stochastic control formulation in (5.9) we reversed the time.

It was shown in [23] and Lions [24] that

$$
\begin{gathered}
u^{\epsilon}(x, 0)=\inf \left\{\int_{0}^{T} \int_{\mathbb{R}^{d}}\left[H^{\star}\left(a, \frac{x}{\epsilon}\right) m+\mathcal{F}\left(\frac{x}{\epsilon}, m\right)\right] d x d t+\int_{\mathbb{R}^{d}} u_{T}(x) m(x, T) d x:\right. \\
\left.\partial_{t} m-\epsilon \Delta m+\operatorname{div}[a m]=0 m(\cdot, 0)=m_{0}\right\},
\end{gathered}
$$

where $H^{\star}$ is the convex conjugate of $H$.

The variational formula for $u^{\epsilon}$ is amenable to the $\Gamma$-convergence techniques, which yield a homogenized limit $\bar{u}$ for the $u^{\epsilon}$, which also satisfies, for an effective Hamiltonian $\bar{H}$, the variational formula

$$
\begin{aligned}
& \bar{u}(x, 0)=\inf \left\{\int_{0}^{T} \int_{\mathbb{R}^{d}} \bar{H}^{\star}(a, m) d x d t\right.+\int_{\mathbb{R}^{d}} u_{T}(x) m(x, T) d x: \\
&\left.\partial_{t} m-\Delta m+\operatorname{div}[a m]=0 m(\cdot, 0)=m_{0}\right\},
\end{aligned}
$$

The limit $\bar{u}$ should also satisfy the first of the two equations of (1.10) and, of course, the question is if (1.12) holds or not. This is equivalent to checking if the limiting variational formula corresponds to a potential-type mfg.

It is known (see [24]), however, that for a mfg to have a potential formulation, the Hamiltonian must be of separated form, that is we must have that

$$
\bar{H}(p, m)=\bar{H}(p)+\bar{F}(m) .
$$

It is a classical fact that the $\Gamma$-limit of variational problems with Lagrangians corresponding to separated Hamiltonians does not give, in general, rise to separated effective Hamiltonians; see, for example, [11]. Hence, the homogenized system is not, in general, of mfg-type.

The last item we discuss here is the rigorous convergence of the solution $\left(u^{\epsilon}, m^{\epsilon}\right)$ of (1.1) when the initial and terminal conditions satisfy (1.13). This is, however, a classical fact in the homogenization theory, where now 
the ansatz (1.7) can be shown to hold rigorously as above, given that, as follows form a simple calculation, the solution of homogenized system (1.10) with initial and terminal conditions as in (1.13) is given by

$$
\bar{u}(x, t)=(p, x)-t \bar{H}(p, 1) \text { and } \bar{m} \equiv 1 .
$$

\section{REFERENCES}

1. Yves Achdou, Francisco J. Buera, Jean-Michel Lasry, Pierre-Louis Lions, and Benjamin Moll, Partial differential equation models in macroeconomics, Philos. Trans. R. Soc. Lond. Ser. A Math. Phys. Eng. Sci. 372 (2014), no. 2028, 20130397, 19. MR 3268061

2. Yves Achdou, Pierre-Noel Giraud, Jean-Michel Lasry, and Pierre-Louis Lions, A long-term mathematical model for mining industries, Appl. Math. Optim. 74 (2016), no. 3, 579-618. MR 3575616

3. Grégoire Allaire, Homogenization and two-scale convergence, SIAM Journal on Mathematical Analysis 23 (1992), no. 6, 1482-1518.

4. Pierre Cardaliaguet, Francois Delarue, Jean-Michel Lasry, and Pierre-Louis Lions, The master equation and the convergence problem in mean field games, ArXiv e-prints (2015).

5. Pierre Cardaliaguet, Jean-Michel Lasry, Pierre-Louis Lions, and Alessio Porretta, Long time average of mean field games, Netw. Heterog. Media 7 (2012), no. 2, 279-301. MR 2928380

6. __ Long time average of mean field games with a nonlocal coupling, Netw. Heterog. Media 7 (2012), no. 2, 279-301. MR 2928380

7. René Carmona and François Delarue, Probabilistic analysis of mean-field games, SIAM J. Control Optim. 51 (2013), no. 4, 2705-2734. MR 3072222

8. Rene Carmona and Francois Delarue, Probabilistic theory of mean field games with applications i, 2018.

9. _ Probabilistic theory of mean field games with applications ii, 2018.

10. Annalisa Cesaroni, Nicolas Dirr, and Claudio Marchi, Homogenization of a mean field game system in the small noise limit, SIAM Journal on Mathematical Analysis 48 (2016), no. 4, 2701-2729.

11. Gianni Dal Maso, An introduction to $\gamma$-convergence, vol. 8, Springer Science \& Business Media, 2012.

12. Xavier Gabaix, Jean-Michel Lasry, Pierre-Louis Lions, and Benjamin Moll, The dynamics of inequality, Econometrica 84 (2016), no. 6, 2071-2111, Appendix G with Zhaonan Qu, Appendix G appearing in the supplementary materials not found within the manuscript. MR 3580263

13. Thierry Goudon and Frédéric Poupaud, Homogenization of transport equations: weak mean field approximation, SIAM J. Math. Anal. 36 (2004/05), no. 3, 856-881. MR 2111918

14. Olivier Guéant, Jean-Michel Lasry, and Pierre-Louis Lions, Mean field games and applications, Paris-Princeton Lectures on Mathematical Finance 2010, Lecture Notes in Math., vol. 2003, Springer, Berlin, 2011, pp. 205-266. MR 2762362

15. Minyi Huang, Peter E. Caines, and Roland P. Malhamé, An invariance principle in large population stochastic dynamic games, J. Syst. Sci. Complex. 20 (2007), no. 2, 162-172. MR 2344101

16. __ Large-population cost-coupled LQG problems with nonuniform agents: individual-mass behavior and decentralized $\epsilon$-Nash equilibria, IEEE Trans. Automat. Control 52 (2007), no. 9, 1560-1571. MR 2352434

17. 55 (2010), no. 12, 2799-2805. MR 2767144

18. Minyi Huang, Roland P. Malhamé, and Peter E. Caines, Nash equilibria for large-population linear stochastic systems of weakly coupled agents, Analysis, control and optimization of complex dynamic systems, GERAD 25th Anniv. Ser., vol. 4, Springer, New York, 2005, pp. 215-252. MR 2143444

19. L Large population stochastic dynamic games: closed-loop McKean-Vlasov systems and the Nash certainty equivalence principle, Commun. Inf. Syst. 6 (2006), no. 3, 221-251. MR 2346927

20. Aimé Lachapelle, Jean-Michel Lasry, Charles-Albert Lehalle, and Pierre-Louis Lions, Efficiency of the price formation process in presence of high frequency participants: a mean field game analysis, Math. Financ. Econ. 10 (2016), no. 3, 223-262. MR 3500451

21. Jean-Michel Lasry and Pierre-Louis Lions, Jeux à champ moyen. I. Le cas stationnaire, C. R. Math. Acad. Sci. Paris 343 (2006), no. 9, 619-625. MR 2269875

22. __ Jeux à champ moyen. II. Horizon fini et contrôle optimal, C. R. Math. Acad. Sci. Paris 343 (2006), no. 10, 679-684. MR 2271747

23. _ Mean field games, Jpn. J. Math. 2 (2007), no. 1, 229-260. MR 2295621

24. Pierre-Louis Lions, Mean field games, Cours de Collège de France. 
25. Pierre-Louis Lions and Jean-Michel Lasry, Large investor trading impacts on volatility, Ann. Inst. H. Poincaré Anal. Non Linéaire 24 (2007), no. 2, 311-323. MR 2310697

26. Pierre-Louis Lions, George Papanicolaou, and S.R.S. Varadhan, Homogenization for hamilton-jacobi equations, preprint.

27. Pierre-Louis Lions and Panagiotis E. Souganidis, Homogenization of degenerate second-order PDE in periodic and almost periodic environments and applications, Ann. Inst. H. Poincaré Anal. Non Linéaire 22 (2005), no. 5, 667-677. MR 2171996

28. Gabriel Nguetseng, A general convergence result for a functional related to the theory of homogenization, SIAM Journal on Mathematical Analysis 20 (1989), no. 3, 608-623.

$\left({ }^{1}\right)$ Collège de France, 11 Place Marcelin Berthelot, 75005 Paris, and CEREMADE, Université de ParisDauphine, Place du Maréchal de Lattre de Tassigny, 75016 Paris, FRANCE email: lions@ceremade.dauphine.fr

$\left(^{2}\right)$ Department of Mathematics University of Chicago, 5734 S. University Ave., Chicago, IL 60637, USA, email: souganidis@math.uchicago.edu 